\title{
Knowledge base in system of value-oriented management of innovative projects
}

\author{
Artemy G. Varzhapetyan \\ Department of Quality Management of Education \\ Saint-Petersburg State University of Aerospace \\ Instrumentation \\ Saint-Petersburg, Russian Federation
}

\author{
Elena G. Semenova \\ Institute of Innovation and Master Basic Training \\ Saint-Petersburg State University of Aerospace \\ Instrumentation
}

\author{
Remir I. Solnitsev \\ Department of Computer Aided Design Systems \\ Saint Petersburg State Electrotechnical University "LETI" \\ Saint-Petersburg, Russian Federation \\ Alena V. Fomina \\ JSC "Central Research Institute of Economy Management \\ and Information Systems "Electronics" \\ Moscow, Russian Federation
}

\author{
Viktor M. Balashov \\ JSC «Scientific and Production Enterprise "Radar MMS"» \\ Saint-Petersburg, Russian Federation
}

\begin{abstract}
Objectives to improve the effectiveness of innovative organizations can be contributed to the introduction of valuebased management. Given there is a huge amount of heterogeneous information typical for such companies, it is advisable to apply ontological engineering methods when constructing the proposed system. The chosen approach makes it possible to create a knowledge base, the use of which helps decision-makers to effectively manage the organization when implementing innovative projects. In the previously proposed dynamic model of managing the results of the organization's activities, the authors used a knowledge base built on the basis of $\mathbf{N}^{3}$ information (non-complete, non-clear, non-numerical), which makes it more difficult to function. The application of ontological engineering methods allows eliminating the identified shortcomings of this model. As a result of the study, the principles of constructing a knowledge base using ontological engineering tools were developed, the algorithms for the functioning of a valueoriented management system based on it were proposed, and an approach to improving the efficiency of an organization using an estimate of the components of the integral consumer value was described. The proposed system of value-oriented management is built in accordance with the ISO 55000 standard and can find its application in innovative organizations.
\end{abstract}

Keywords-value-oriented management; knowledge base; ontological engineering.

\section{INTRODUCTION}

The specific nature of the activity of an innovation organization initially assumes a high level of risks, namely in connection with the need to introduce such technologies and approaches that have not been used anywhere else. In such circumstances, the person who makes decisions on which the management of the entire organization is built has a huge responsibility, but for objective reasons, he or she cannot rely on the practical experience that his/her colleagues have developed. The only way to minimize risks is to constantly monitor the economic performance of the organization and analyze the results of earlier decisions. Such approach will allow, if necessary, promptly adjusting the management policy. At the same time, it is obvious that a continuous analysis of the organization's performance results in the processing of significant volumes of heterogeneous information, the volumes of which are replenished in real time. To facilitate the task of improving the effectiveness of the decision-maker, a valueoriented results-based management system should be introduced at innovation enterprises [1], the work of which will be built on the basis of the knowledge base created with the ontological engineering tools.

In the model previously developed by the authors, the questions of functioning of the knowledge base built on the basis of $\mathrm{N}^{3}$ information (non-complete, non-clear, nonnumerical) have not been fully worked out, which makes it difficult to process and analyze it for subsequent decisionmaking. In order to improve it, the authors decided to apply the methods of ontological engineering, suggesting that such approach should significantly facilitate the work with significant amounts of heterogeneous information necessary for making managerial decisions aimed at improving the efficiency of an innovative organization.

In the course of work on the study, the authors set themselves the following tasks: to develop algorithms for the functioning of a value-oriented management system with the use of a knowledge base built by means of ontological engineering; to identify opportunities to increase the efficiency of high-tech organizations as a result of the introduction of a value-oriented management system with an improved knowledge base. 


\section{LITERATURE REVIEW}

Theoretical formation of the concept of value-oriented management refers to the turn of the 1980s-1990s. The first mentions of management by values appeared in the 1980s in the monograph of Peters and Waterman (1984), who proposed the "McKinsey 7s" model. In the years of the establishment of the value-oriented system (VOS), the publications were devoted primarily to the economic and financial components of value [2]. With the penetration into the management of the ideas of axiology (the general theory of value), the study of its intangible components began. Interesting are the works of Majer [3], who identified the basic principles of VOS and gave recommendations on the introduction of value management in companies, PMBoK 6 [4] and the Japanese standard P2M [5]. Among publications in Russian, it is necessary to note the monograph of Prigozhin [6], generalizing the ideas of earlier publications, as well as the works of Bushuev [7] and Grigoryan [8], devoted to the harmonization of the interests of the parties engaged in the implementation of an innovative project. The management of complex high-tech products was previously considered by the authors of this article as well $[9,10,11]$.

The definition of ontology in technical applications was first given by Gruber [12]: ontology is a specification of conceptualization (a structure of reality considered independently of the vocabulary of the subject domain and a particular situation). In 1996, the work of Japanese scientists Mizoguchi and Ikeda was published [13], who proposed a new research field - "ontological engineering", the goal being to provide a basis for constructing ontological models. The standard IDEF5 [14], which appeared in the late 1990s, allows representing data obtained by processing ontological queries in a simple, natural graphical form. Gomez-Perez [15] considered the application of ontologies in knowledge management. Casellas [16] gave a detailed analysis of various methods of ontological engineering. Gavrilova [17] deals with the application of ontological engineering in Russia. The use of thesauri in ontological engineering is the subject of a monograph by Dobrov et al. [18].

At the same time, the management of an organization must be built in accordance with the accepted standards. ISO 55000 [19] suggests achieving the organization's goals, a balance between costs, prospects, risks and ensuring the required asset performance. ISO 9001 [20] is aimed at improving management based on modern information systems, enhancing efficiency and cost-effectiveness. ISO 9004 [21] is dedicated to management in order to achieve sustainable development of the organization in a complex and constantly changing environment, where innovation is one of the most important values.

The literature practically does not cover the methods of VOS as a dynamic system using ontological engineering tools for managing the development of projects for high-tech industries; therefore, the authors consider it reasonable to propose a method for managing results precisely when planning complex innovative projects.

\section{METHODOLOGY}

When writing this paper, the authors based on the dynamic model of managing the results of the organization's activities specialized in complex high-tech products described in their previous works $[9,10,11]$ and being a multidimensional, multiply connected and multimodal value-oriented results management system (VORMS) functioning through a knowledge base that is built on the basis of $\mathrm{N}^{3}$ information (non-complete, non-clear, non-numerical). Processing and analysis of such information for subsequent decision-making is greatly facilitated if ontological engineering methods are applied.

The control system under consideration is built in accordance with the ISO 55000 [19] standard and is intended for monitoring the state of dynamic processes characterized by continuity and discreteness. The functioning of the system assumes that it is possible to repeat earlier procedures and operations with the new data added.

Earlier works by the authors [1] contain the rationale for the benefits of results management, which was chosen to build the structure of the value-oriented management system, in comparison with the management by goals.

\section{RESULTS}

\section{Structure of VORMS}

The selected VORMS structure with feedback loops is shown in Fig. 1.

An approach to the application of ontological engineering for the construction of VORMS is presented in Fig. 2, where: $X_{\Sigma}$ - initial order and resource data, $Y_{\Sigma}$ - output product, $W_{i j}-$ operators of transformation of flows of resources and information with the inclusion of measuring elements:

$$
\begin{gathered}
W_{i j}=\frac{a_{i j} p^{2}+b_{i j} p+c_{i j}}{\alpha_{i j} p^{2}+\beta_{i j} p+\gamma_{i j}} p-p \tau_{i j}, \\
i, j=\overline{1, n}, \overline{1, m}
\end{gathered}
$$

$\Phi_{i j}(p)$ - control operators with the inclusion of actuators. 


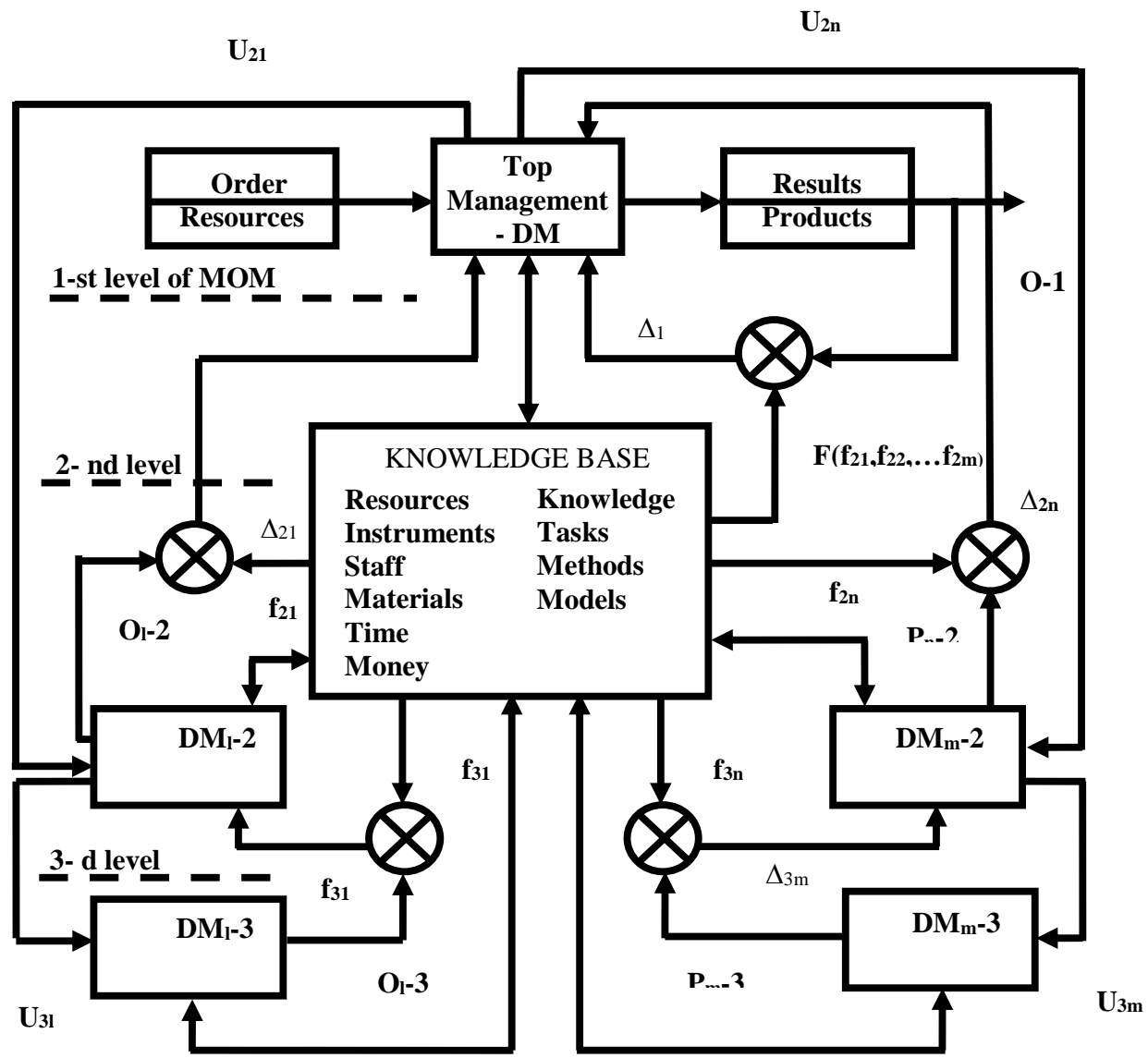

Fig. 1. Approximate structure of VORMS (developed by the authors)

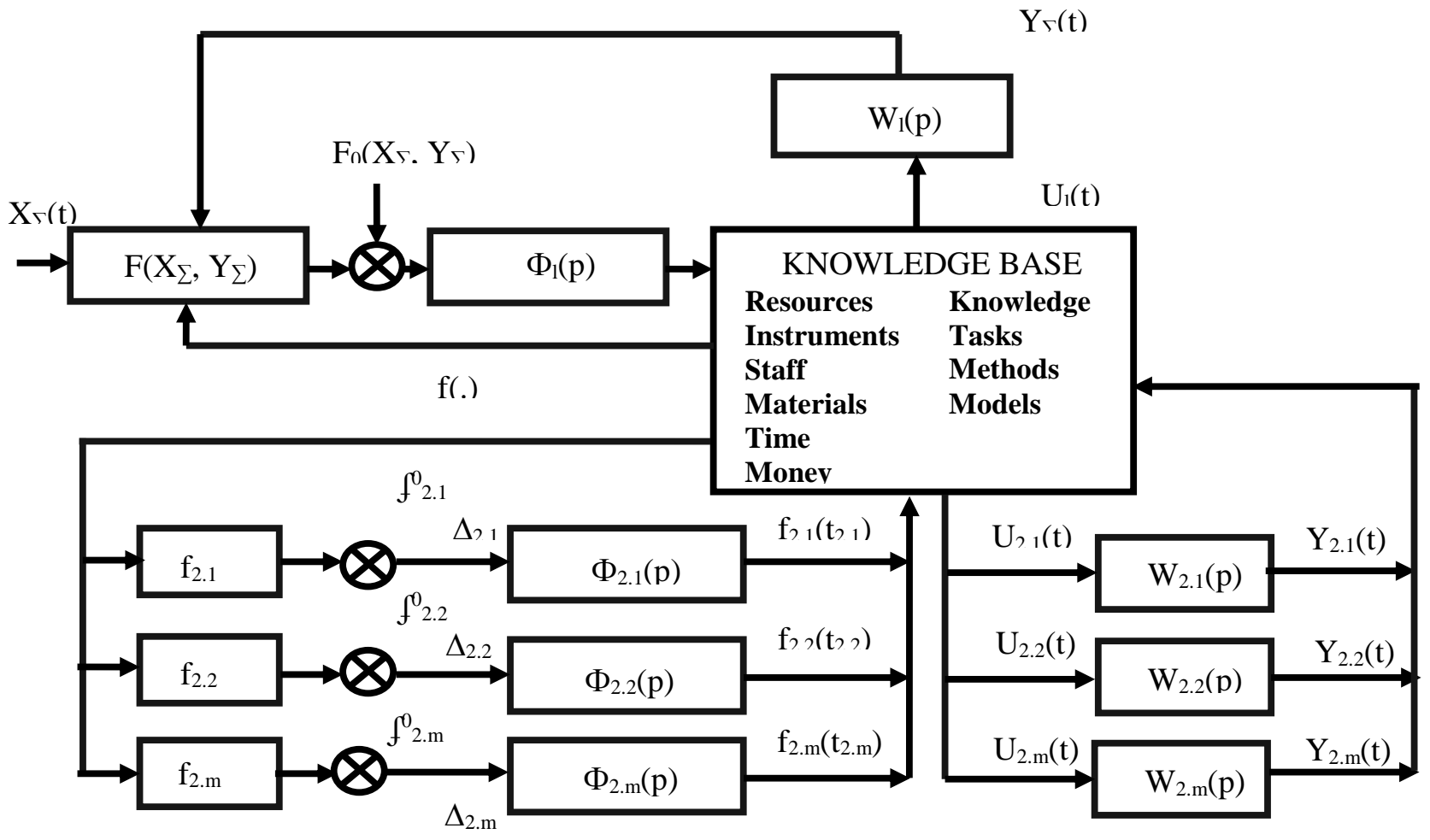

Fig. 2. Application of ontological engineering in VORMS (developed by the authors) 
The parameters $a_{i j}, b_{i j}, c_{i j}, \tau_{i j}, \alpha_{i j}, \beta_{i j}, \gamma_{i j}, i, j=\overline{1 \div n, m}$, are calculated after processing of static data on input and output material and information flows.

The results $P-1, P_{2.1} \ldots P_{3 . n}$ are assessed using the functional $P-1=F\left(Y_{\Sigma}, X_{\Sigma}\right)$, where $Y_{\Sigma}-$ production vector, $X_{\Sigma}-$ the organization's resource cost vector.

Respectively, the results $P-2, P-3$ and possibly of the next levels will be:

$$
\begin{aligned}
& P_{2.1}=f_{2.1}\left(Y_{2.1}, X_{2.1}\right) \ldots P_{2 . n}=f_{2 . n}\left(Y_{2 . n}, X_{2 . n}\right), \\
& P_{3.1}=f_{3.1}\left(Y_{3.1}, X_{3.1}\right) \ldots P_{3 . m}=f_{3 . m}\left(Y_{3 . m}, X_{3 . m}\right) .
\end{aligned}
$$

Restrictions are entered as:

$$
Y_{\Sigma}^{\min } \leq Y_{\Sigma} \leq Y_{\Sigma}^{\max }, X_{\Sigma}^{\min } \leq X_{\Sigma} \leq X_{\Sigma}^{\max }
$$

Respectively:

$$
\begin{aligned}
& Y_{2.1}^{\min } \leq Y_{2.1} \leq Y_{2.1}^{\max } \ldots \leq Y_{2 . n} \\
& \leq X_{2.1} \leq \cdots \leq X_{2 . n} \\
& Y_{3.1}^{\min } \leq Y_{3.1} \leq Y_{3.1}^{\max } \ldots \leq Y_{3 . n}
\end{aligned}
$$

Assessments are made according to accepted norms $\left\|Y_{i j}\right\|$, $\left\|X_{i j}\right\|$.

The estimates (4) and (5-7) are coordinated through controls $U_{2.1} \ldots U_{2 . n} ; U_{3.1} \ldots U_{3 . n}$, representing the choice of the decisionmaker's decisions on tasks and resources. The choice of the solution is based on the iterative algorithm considered below.

$$
\begin{aligned}
& U_{2.1}=K_{2.1}^{0} \cdot \Delta_{2.1}+K_{2.1}^{1} \cdot \Delta_{2.1}^{\prime}+K_{2.1}^{2} \cdot \int \Delta_{2.1} \\
& U_{3 . m}=K_{3 . m}^{0} \cdot \Delta_{3 . m}+K_{3 . m}^{1} \cdot \Delta_{3 . m}^{\prime}+K_{3 . m}^{2} \cdot \int \Delta_{3 . m}
\end{aligned}
$$

where $\Delta_{2.1}(t) \ldots \Delta_{3 . m}(t)$ - measurable functions of time, $\Delta_{2.1}^{\prime}, \int \Delta_{2.1}, \ldots \Delta_{3 . \mathrm{m}}^{\prime}, \int \Delta_{3 . \mathrm{m}}-$ derivatives and integrals of these functions.

The coefficients $K_{i j}, i, j=\overline{n, m}$ in (9) are selected based on the iterative algorithm considered below.

\section{Formation of knowledge and controls base}

The formation of a knowledge base by means of ontological engineering can be represented in the form of a tuple $R=<R_{l}$, $R_{2}, R_{3}, R_{4}, F>$, where $R_{l}$ - the organization's subject domain, $R_{2}$ - the organization's structure, $R_{3}$ - relationships between ontology components, $R_{4}-$ multitude of the subject domain's notions, $F$ - terms identification function. $R_{3}-$ multitude of relationships connecting elements of the multitude $R_{l}$ with ontologies $R_{2}$ (binary relationships), ontology $R_{4}$ with function $F$ (binary relationships).

$$
F=\left\langle F_{1}, F_{2} \ldots F_{6}\right\rangle \text {, e.g., MP and CLADO units }[10,11] \text { : }
$$

$F_{1}$ "Dossier" - providing employees with rights and responsibilities, resources;

$F_{2}$ "Notebook" - reports on the current, planned, implemented tasks, contracts;

$F_{3}$ "Mode" - detailed planning in the production of results, etc.

In the course of VORMS dynamics, inquiries and answers of decision-makers and PICs to the knowledge base are formalized by means of ontological engineering using the function $F$ in the appropriate language (PROLOG, JAVA, etc.) $[22,23]$.

The achievement of the results is verified on the basis of the feedback principle: $\Delta=$ (planned result - actual result $) \leq E$, where $E$ - permissible error.

The decision-maker is forming control as $U=<$ tasks, resources>

$U=F\left(R_{l}(k T), R_{2}(k T)\right), k=0, N, T_{1}<T<T_{2}$.

Formulation of tasks, resources and $T$ must be made by the decision-maker who must foresee $\Delta(t)$ and then input $\Delta^{\prime}(t)$, $\int \Delta(t)$ into the control law.

$\Delta(t)$ comes to the decision-maker via feedback and he/she, in turn, inputs $\Delta^{\prime}(t), \int \Delta(t)$ into control.

\section{Algorithm for correction of the decision-maker's decisions}

$U\left(R_{1}, R_{2}\right)$ is formed using the knowledge base under the following algorithm [9]. The initial data are defined as:

$$
D=\left\{X_{i}=\Phi\left(x_{1}, x_{2}, \ldots, x_{n}\right)\right\}, i=\overline{1, N},
$$

where $D-$ a multitude of possible options for making decisions to control $U$ (expected orders, resources, implementation processes, etc.); $\mathrm{n}-$ the number of characteristics, signs of the $i$-th decision (marketing, social, ergonomic, technical):

$$
K=F\left(k_{1}, \ldots, k_{m}\right),
$$

where $K$ - efficiency index, functional from the criteria of formation $D ; m$ - the number of criteria (cost, resources, ergonomics, technicality).

$$
O=\left(O_{1}, O_{2}, \ldots, O_{r}\right)
$$

where $O-$ the vector of restrictions for the formation of $D$ for a given $K$.

Decision-makers appoint $E_{\mathrm{i}}$ - the utility of particular decisions. $E_{\mathrm{i}}$ is corrected using respective ontological tools via the knowledge base and is reduced to finding $X_{i}, i=1, N$, providing:

$$
\max \{K\}=\max _{i=1}\left\{r_{i}\right\}, r_{i}=f\left(k_{1}, k_{2}, \ldots, k_{n}\right),
$$

where $r_{i}$ - complex convolution of criteria values of one variant, $f$-convolution function.

Functions of $f$ can be constructed by known methods (deterministic, probabilistic, fuzzy logic), the most suitable of which are loaded into the knowledge base. The simplest way is additive $\mathrm{f} \rightarrow \Sigma$ :

$$
r_{i}=\lambda_{1} \bar{K}_{l}+\lambda_{2} \overline{K_{l 2}}+\cdots+\lambda_{m} \overline{K_{l m}}, i=\overline{1, N}
$$

where $\lambda_{l}$-weight coefficients $\sum_{l=1}^{m} \lambda_{l} \leq 1 ; \bar{K}_{l}-$ the relative values determined by the relation:

$$
\begin{aligned}
& \bar{K}_{l}=\frac{K_{\operatorname{maxl}}-K_{l}}{K_{\operatorname{maxl}}-K_{\min l}} \\
& \text { or } \quad \bar{K}_{l}=\frac{K_{l}-K_{\operatorname{minl}}}{K_{\operatorname{maxl}}-K_{\operatorname{minl}}} \\
& \text { where } k_{\operatorname{minl}}<k_{l j}<k_{\operatorname{maxl}} .
\end{aligned}
$$

Depending on the content, $\overline{k_{l j}}$ (value, resources, processes) is taken as (15) or (16). 
For each possible variant of decision $\mathrm{X}_{\mathrm{i}}$, the criteria $\overline{\mathrm{K}_{\mathrm{lj}}}$ are filled with specific numerical values from the knowledge base $\mathrm{i}=\overline{1, \mathrm{~N}}, \mathrm{j}=\overline{1, \mathrm{n}}$. Meantime, from the knowledge base the data $K_{\max } i, K_{\min } I$ are found. The weight coefficients $\lambda_{j}$ are determined by the following algorithm, which is input in the knowledge base.

The algorithm is based on the heuristic ranking of decisionmakers of decisions by utility:

$$
\left\{\begin{array}{c}
\lambda_{1} \overline{k_{11}}+\lambda_{2} \overline{k_{12}}+\cdots+\lambda_{m} \overline{k_{l m}}=E_{1} \\
\cdots \cdots \cdots \cdots \cdots \cdots \cdots \cdots \cdots \cdots \cdots \cdots \cdots \cdots \cdots \cdots \cdots \cdots \cdots \cdots \cdots \cdots \cdots \cdots \\
\lambda_{1} \overline{k_{N 1}}+\lambda_{2} \overline{k_{N 2}}+\cdots+\lambda_{m} \overline{k_{N m}}=E_{N}
\end{array}\right.
$$

where $E_{1}, \ldots, E_{N}-$ utilities of decisions $\left.\left.\left.E_{1}\right\} E_{2}\right\} \ldots\right\} E_{N}, N-$ the number of decision variants; $m$ - the number of criteria; $\lambda_{i}$ weight coefficients; $k_{i, l}$ - the $l$-th criterion of the $i$-th variant.

As a result, a matrix is formed from the numerical values of the criteria:

$$
\left|\begin{array}{ccc}
\overline{k_{11}} & \overline{k_{12}} & \overline{k_{1 m}} \\
\cdots & \ldots & \ldots \\
\overline{k_{N 1}} & \overline{k_{N 2}} & \overline{k_{N m}}
\end{array}\right|
$$

where $i=\overline{1, N}-$ decision variants, $j=\overline{1, m}-$ criteria depending on characteristics of $x_{s}$ in (10).

Decision-makers appoint numeric values of utility $E_{i}$, which are normed in compliance with the condition $\prod_{i=1}^{N} E_{1}^{0} \leq 1$. Hence, from (17) the desired $\lambda_{\mathrm{j}}$ can be found:

$$
\left\{\begin{array}{c}
\lambda_{1} \overline{k_{11}}+\lambda_{2} \overline{k_{12}}+\cdots+\lambda_{m} \overline{k_{l m}}=E_{1}{ }^{0} \\
\cdots \cdots \cdots \cdots \cdots \cdots \cdots \cdots \cdots \cdots \cdots \cdots \cdots \cdots \cdots \cdots \cdots \cdots \cdots \cdots \\
\lambda_{1} \overline{k_{N 1}}+\lambda_{2} \overline{k_{N 2}}+\cdots+\lambda_{m} \overline{k_{N m}}=E_{N}{ }^{0} \\
\prod_{j=1}^{m} \lambda_{j} \leq 1 \\
\prod_{i=1}^{N} E_{1}^{0} \leq 1 .
\end{array}\right.
$$

The system of algebraic equations (19) is solved with respect to $\lambda_{\mathrm{j}}$. Convolutions are formed in the form (14) or in a more balanced form:

$$
r_{i}=\sum_{j=1}^{m}\left(\frac{\lambda_{j} \overline{K_{\iota \jmath}}}{c_{i} / c_{\max }}\right)
$$

where $c_{i} / c_{\max }-$ multiplier of the ratio of the cost of the $i$-th option to the maximum value $C_{\max }=\max _{1 \leq i \leq N}\left\{C_{i}\right\}$. The problem (13) is solved in the knowledge base by the procedure given for finding the extremum [24].

By the values received of $r_{i}$ in compliance with the functional (13), but with weight coefficients already $\lambda_{j}$ the estimated values of utility $E_{i}$ are found.

In special cases of extremum search (gully situation), special algorithms are applied [25].

The $i$-th decision is chosen, corresponding to $\max _{1 \leq i \leq N}\left\{E_{i}\right\}$, and a discrepancy is compiled $\Delta=\left|\overline{E_{l}{ }^{0}}-E_{i}\right|$. If $\Delta \leq \delta_{\text {add }}$, the process is finished; if not, the next iteration is made.

\section{DISCUSSION}

In the context of globalization and transformations, the knowledge potential management is an effective tool for increasing the effectiveness of organizations [26]. The application of ontological engineering methods in the system of value-oriented management makes it possible to create a knowledge base for the integrated accounting of work results. This knowledge base is in fact the center for managing the tree of results achieved by an innovation organization, including individual units in its structure and PICs. Accounting for these components in the knowledge base allows one to assess their quality and impact on the overall performance of an innovative organization.

Within the chosen approach, the company's activity is viewed as the result of implementation of individual instruments, as well as various procedures and operations. As source data, the knowledge base is filled with information about the resources of the enterprise, as well as the criteria and restrictions in connection with obtaining the main products of the enterprise. In the process of their analysis, the decisionmaker receives numerous feedbacks, enabling ultimately to more effectively control the basic processes, characteristic of an innovation organization, including design, production, sales, marketing.

If in previous works the issues of functioning of the knowledge base have not been fully worked out, in this paper the principles of its formation are proposed, supplemented with recommendations for its use, which is an important step for increasing the effectiveness of organizations in implementing innovative projects. Unlike previous works, the approach proposed in this paper assumes that individual components of value are formed in the form of concrete management results.

The proposed system allows accounting for the influence of the human factor, taking into account the motivation of each employee in getting the results. When implementing the system, it is recommended to set goals for each employee: both long-term (career growth in 5-10 years) and intermediate (key results during the year). Thus, each employee understands his/her opportunities and possible ways of qualification growth, and his/her motivation is confirmed by "milestones", when the set of tasks facing an employee is determined taking into account his/her abilities and desires. To create confidence in the correctness of the overall goal, publicity, transparency and complete informativeness of the decision-maker-1 and, within the appropriate limits, DMi-2, DMj-3, i = 1, n; $\mathrm{j}=1, \mathrm{~m}$ should be provided. It is recommended to encourage good results and take steps to overcome the problems identified.

The knowledge base, built on the basis of ontological engineering, allows introducing the elements of automated management in decision-making. On the one hand, this approach makes it much easier to process and analyze information about the performance of the enterprise as a whole and its particular units. The decision-maker focuses on the answer from the knowledge base, which takes into account the resources, criteria and limitations of the company as much as possible. The chosen approach allows avoiding qualitative and in many respects quantitative mistakes in building a management system, which is especially important in an innovative organization for which a high level of risk is initially characteristic.

On the other hand, each automated system, including those built using ontological engineering, has its limitations, which are laid when selecting the source data and the algorithm for their processing, and then influencing the result obtained from 
it. Although the proposed decision-making system presupposes the consideration of staff motivation, it remains questionable whether it can completely eliminate the influence of the human factor. The coefficients in assessing the utility of certain decisions are appointed by the decision-maker, while even a well-balanced management policy may not lead to the desired results due to the unfairness of individual performers.

The knowledge base, built on the basis of ontological engineering, can be used to create a value-based management system to reduce risks and improve the efficiency of the organization, but it requires individual adaptation to the needs of a particular project [27]. The choice of software to ensure the functioning of the proposed knowledge base requires a further study. According to the authors of the paper, this role can be performed by the CLADO system with its MP (Management Planning) subsystem [10]. Its adaptation to build an improved knowledge base using ontological engineering tools will be the topic of the further research.

\section{CONCLUSION}

Organizations, the activities of which are related to the introduction of previously applied technologies and the implementation of innovative projects require a special approach to their management: there are no ready solutions in this area and one can only focus on own experience. To improve the efficiency of these enterprises and reduce the level of risks that are known to be high, it is advisable to use a value-oriented management system. Adopting a balanced management decision requires taking into account a huge amount of input data, including resources, criteria and limitations of an innovation organization, while this information is being accumulated in real time. The formation of a knowledge base using ontological engineering tools on its basis seems to be the most effective approach for analyzing and subsequently making managerial decisions.

The created system is designed to provide prompt correction of the management policy of an innovative organization in the course of implementation of high-tech projects, taking into account numerous incoming feedback from particular units and PICs. The developed approach is designed to improve the effectiveness of decisions, and thus the effectiveness of the entire enterprise, as well as to reduce the impact of the human factor.

The knowledge base, built using ontological engineering tools, has the prospects of being introduced in the operation of innovative organizations; however, in each specific case it needs to be finalized taking into account the features of the project and choosing the optimal software. Each enterprise has its own set of resources, criteria and restrictions that are in a certain relationship with each other, and the adaptation of the proposed database to its individual needs may include the improvement of the functioning algorithms and the principles of knowledge base organization, selection of optimal ontological engineering tools, development of specific mathematical models of dynamics management of the organization accounting for its characteristics.

\section{References}

[1] Yu.A. Antokhina, A.G., Varzhapetyan, and E.G. Semenova, "Valueoriented management of innovative projects," Questions of Radio Electronics, vol. 5, pp. 54-61, 2017.

[2] A. Rappaport, "Corporate performance standards and shareholder value," Journal of Business Strategy, vol. 3, no. 4, pp. 37-49, 1983.

[3] K. Majer, Values-Based Leadership: A Revolutionary Approach to Business Success and Personal Prosperity. San Diego, California: MajerCommunications, 2004

[4] PMBOK, A Guide to the Project Management Body of Knowledge, 6th ed. Project Management Institute, 2017.

[5] P2M, A Guidebook of Project and Program Management for Enterprise Innovation. Japan PMAJ, 2008.

[6] A.I. Prigozhin, Goals and Values. New Methods of Working with the Future. Moscow: Delo, 2010

[7] S.D. Bushuev, and N.S. Bushueva, "The mechanisms of value formation in the activities of project-oriented enterprises," Eastern-European Journal of Enterprise Technologies, no. 1/2 (43), pp. 118-126, 2010.

[8] T.G. Grigoryan, "Perfection of models of value-based management of project portfolios," Eastern-European Journal of Enterprise Technologies, no. 3(74), pp. 43-49, 2015.

[9] R.I. Solnitsev, "Information technologies in instrument making," Scientific Instrumentation, vol. 11, pp. 80-83, 2001.

[10] R.I. Solnitsev, "Outcomes Management System - "CLADO", in Proceedings of the International Conference "Practice of Russian Comprehensive Model of Task and Resource Management, St. Petersburg: GUAP, pp. 18-27, 2014.

[11] Yu.A. Antokhina, R.I. Solnitsev, and A.G Varzhapetian, "Improving the quality of the functioning of the organization with outcomes management," Economics and Management, no. 11, pp. 56-63, 2016.

[12] T.R. Gruber, "A translation approach to portable ontologies," Knowledge Acquisition, vol. 5, no. 2, pp. 199-220, 1993.

[13] R. Mizoguchi, and M. Ikeda, Towards Ontology Engineering. Technical Report AI-TR-96-1/I.S.I.R. Osaka: Osaka University, 1996, pp. 86-108.

[14] IDEF5 - Ontology Description Capture Method, 1998.

[15] A. Gomez-Perez, Ontological Engineering: With Examples from the Areas of Knowledge Management, e-Commerce and the Semantic Web. London: Springer, 2004.

[16] N. Casellas, Legal Ontology Engineering. Springer, 2011.

[17] T.A. Gavrilova, and D.I. Muromtsev, Intellectual Technologies in Management: Tools and Systems, 2nd ed. St. Petersburg: Vysshaya shkola menedzhmenta, 2008.

[18] B.V. Dobrov, V.V. Ivanov, N.V. Lukashevich, and V.D. Solovev, Ontologies and Thesauruses. Models, Tools, Applications: Tutorial. Moscow: Internet-Universitet Informatsionnykh Tekhnologii; BINOM. Laboratoriya znanii, 2009.

[19] ISO 55000:2014. Asset Management. Overview, Principles and Terminology, 2014.

[20] ISO 9001:2015. Quality Management Systems - Requirements, 2015.

[21] ISO 9004:2009. Managing for the Sustained Success of an Organization. A Quality Management Approach, 2009.

[22] Stanford Center for Biomedical Informatics Research. http://protege.stonford.edu

[23] TopQuadrant. http://topbraidcomposer.com

[24] Control System Toolbox, http://www.methworks.com//products/Confril.html

[25] I.G. Chernorutskii, Optimal Parametric Synthesis. Leningrad: Energoatomizdat, 1987.

[26] J. Raudeliūnienè, V. Davidavičienè, and A. Jakubavičius, "Knowledge management process model," Entrepreneurship and Sustainability Issues, vol. 5, no. 3, pp. 542-554, 2018. https://doi.org/10.9770/jesi.2018.5.3(10)

[27] V.E. Guseva, L. Ungureanu, and E.A. Kuzmin, "Mathematical models of uncertainty in economics," Journal of Applied Economic Sciences, vol. XII, no. 3(49), pp. 753-765, 2017. 